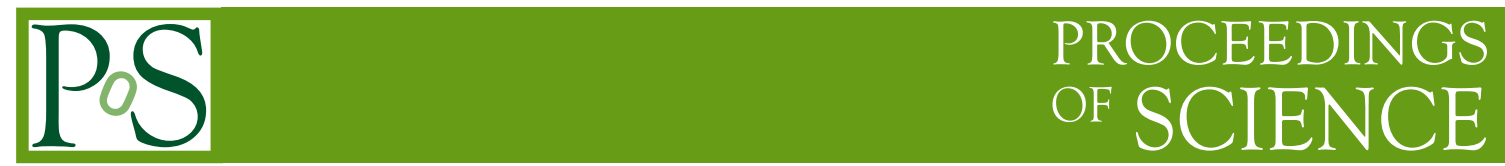

\title{
The story of Majorana's exchange force
}

\author{
David Brink* \\ Department of Physics \\ Rudolf Peierls Institute for Theoretical Physics, 1 Keble Road, Oxford, UK \\ E-mail: thph0032@herald.ox.ac.uk
}

\begin{abstract}
Soon after the discovery of the neutron Heisenberg published a theory of the structure of nuclei based on exchange forces between nucleons which had some very nice features. In January 1933 Majorana came to Leipzig with a fellowship to spend several months in Heisenberg's Institute. On arrival Majorana pointed out two weak points in Heisenberg's theory. It failed to account for the strong binding of the alpha particle and it required a strongly repulsive core in the nuclear force to avoid nuclear collapse. Majorana proposed a new kind of exchange force which avoided these difficulties and wrote a paper which was published at the beginning of March. His theory was so satisfying aesthetically that it was accepted immediately. This lecture will analyze the origin, motivation and impact of Majorana's theory and give some information about his stay in Leipzig.
\end{abstract}

International Conference - Ettore Majorana's legacy and the Physics of the XXI century October 5-6 2006

University of Catania, Italy

${ }^{*}$ Speaker. 


\section{Introduction}

In 1931 nuclear structure was hardly understood. In the introduction to his book 'Atomic nuclei and radioactivity' Gamow [1] wrote that "In accordance with concepts of modern physics we assume that all nuclei are built up of elementary particles -protons and electrons". He assumed also that one of the protons and electrons were bound into alpha particles. He goes on to point out some of the difficulties of this model and later in the introduction he said " The usual ideas of quantum mechanics absolutely fail in describing the behaviour of nuclear electrons." He decided not to be distracted by this problem "For some unknown reason, although the electrons in the nucleus behave in a peculiar and obscure way, this does not affect very much the laws governing the motion of the nuclear $\alpha$-particles and protons; we can treat nuclear processes involving only $\alpha$-particles and protons independently of the presence of the nuclear electrons.

The situation changed dramatically with the experiments of Joliot-Curie which culminated in the discovery of neutron by Chadwick in Feb 1932. Very soon there were suggestions that neutrons were something like protons. In April 1933 Ivanenko wrote "The chief point of interest is how far neutrons can be considered as elementary particles (something like protons and electrons)."

In a series of three papers published in 1932 Heisenberg put forward a number of ideas which formed the basis for later investigations of nuclear forces and nuclear structure. He suggested that nuclei were composed of protons and neutrons and that nuclear structure could be described by the laws of quantum mechanics in terms of the interaction between the nuclear particles.

News of the Joliot-Curie experiments reached Rome at the beginning of 1932. Majorana's colleagues in Rome recalled that he became interested immediately and guessed that the Paris group had detected the neutron even before the conclusive experiments of Chadwick. He was able to explain the structure of atomic nuclei by introducing an exchange interaction between protons and neutrons. Fermi was attending a conference in Paris in July 1932 and suggested to Majorana that he should make an announcement about his theory. Majorana did not accept this offer. $\mathrm{He}$ insisted that his work was not ready for publication.

After Heisenberg had published his papers in the second half of 1932 Fermi was able to persuade Majorana to go to Leipzig to discuss with Heisenberg. At the end of 1932 Majorana made an application to the CNR (Consiglio Nazionale di Ricerche) for a fellowship to visit Leipzig and also to take the opportunity to attend some meetings in Germany and Denmark. He arrived in Leipzig on 19th January 1933. It seems that Heisenberg was very impressed with Majorana's work on exchange forces and persuaded him to publish it. On 18th of February he wrote to his father that he had finished the paper. It appeared in the Zeitschrift für Physik on 3rd March 1933 [2]. A few weeks later he published an Italian version of the paper in 'La Ricerca Scientifica. This was a requirement for scientists who had made a publication while holding a fellowship from the Consiglio Nazionale delle Ricerche.

Already by 1932 many nuclear masses and mass defects had been measured. When the neutron came on the scene binding energies in the neutron proton model could easily be obtained from the mass defects. The binding energies were rather constant through the periodic table. Nuclear radii had also been estimated and it was known that they could be represented approximately by the formula $R \propto A^{1 / 3}$ where $A$ is the mass number. In Majorana's words nuclei seem to be composed "of some sort of matter with the same properties of size and impenetrability as macroscopic matter. 
Light and heavy nuclei are built up of this matter and the difference between them depends mainly on their content of nuclear matter".

Apart from binding energies and sizes not much else was known about nuclei when Heisenberg and Majorana proposed their theories. The $\alpha$-particle was well known and in 1932 Urey [5] and his collaborators discovered the deuteron. Its spin was not measured until 1934 and the quadrupole moment only in 1939. Both Heisenberg and Majorana assumed that the neutron-proton force was the main interaction between nucleons The idea that $n-n, p-p$ and $n-p$ interactions were approximately equal was not suggested until 1936.

Majorana stayed in Leipzig until August 1933. He made a visit of about a month to Copenhagen in March. There he had the opportunity to meet Niels Bohr and Pauli as well as a number of other prominent theoretical physicists.

\section{Heisenberg's theory}

In June 1932, just four months after Chadwick's results were announced, Heisenberg's first paper [3] was published. He suggested that, in analogy with the structure of the $\mathrm{H}_{2}^{-}$ion, the origin of force between protons and neutrons could be illustrated by imagining the exchange of electrons without spin which obey the laws of Bose-statistics. Heisenberg's paper contains remarkable insights. His model is a precursor to Yukawa's meson exchange theory. It was the first to introduce isospin variables which for Heisenberg were a convenient way to describe the exchange process. In his paper the proton-neutron interaction was the main component of the nuclear force. The idea that p-p, n-n and n-p forces all had the same strength was proposed by Cassen and Condon [6] only in 1936.

But Heisenberg's theory had several problems one of which was the strong spin-dependence of the interaction. The deuteron was discovered by Urey and his collaborators [5] in 1932 and in his first paper Heisenberg discussed the deuteron binding. With his choice of the sign of his interaction the wave function of the deuteron was symmetric in the isospin variables, symmetric in the space coordinate and antisymmetric in spin. Thus Heisenberg's theory predicted that the deuteron spin was $S=0$. This is incorrect, but its spin $S=1$ was not measured until 1934. There was another difficulty which was already apparent in 1933. The theory predicted that the neutronproton interaction was attractive in a state with spin $S=0$ and repulsive when $S=1$. In a many particle system the wave function was symmetrical in the isospin variables and was antisymmetric for exchange of the neutron-proton space-spin variables. Thus the deuteron was a closed shell nucleus and not the alpha particle. Majorana pointed out that Heisenberg's interaction could not account for the large binding of the alpha particle.

Majorana drew attention to another problem with Heisenberg's theory for nuclear matter. Because of the symmetry of the wave function the nuclear binding would become very large for a heavy nucleus for a purely attractive neutron-proton potential. Indeed Heisenberg found that the effective potential $U\left(\mathbf{r}_{1}-\mathbf{r}_{2}\right)$ must be strongly repulsive for small separations in order that the nuclear matter should be stable with a constant density, that is in order that the nuclear force should saturate. 


\section{Majorana's exchange force}

When Majorana arrived in Leipzig he seemed to have a very positive impression of the town and of the Physics Institute. He wrote to his mother on 22 January 1933 that he had already had a long discussion with Heisenberg. Inglis from America was another young visitor. They had met in Rome and Inglis showed him around the town. Majorana and Heisenberg must have discussed the exchange theory of nuclear forces during those first days. Heisenberg's third paper [4] was not published when Majorana arrived in Leipzig. In a footnote he " thanks Professor Heisenberg very much for being able to see his paper before it was published". There must have been very intense discussions between them. Heisenberg recognized the advantages of Majorana's theory and urged him to publish it. The paper was finished quickly and came out just a few weeks later on 3rd March 1933 [2]. In order that Majorana could finish the paper so quickly he must have had the details of his theory worked out when he arrived in Leipzig. He only had to add something about its relation to Heisenberg's theory. This is consistent with the recollections of hs colleagues in Rome.

The first part of the paper discussed Heisenberg's theory and pointed out some of its limitations. Both Majorana and Heisenberg had the same general picture of the structure of nuclei. Majorana wrote: On the contrary it seems as though nuclei consist of rather independent components which interact only on immediate contact, that is a sort of matter with the same properties of size and impenetrability as macroscopic matter. Light and heavy nuclei are built up of this matter and the difference between them depends mainly on their different content of "nuclear matter".

Heisenberg had to assume repulsive forces between neutrons and protons in order to obtain the desired ratio between the number of particles an the nuclear volume. Majorana thought that this was a weakness and wrote that: "Such a solution would be aesthetically unsatisfactory, however, since we would not only have attractive forces of unknown origin between the particles, but also at short distances, repulsive forces of enormous magnitude corresponding to a potential of several million volts".

Another weakness of Heisenberg's interaction was its spin dependence which was attractive for antiparallel spins and repulsive for parallel spins. Majorana wrote that this interaction could not account for the large binding of the $\alpha$-particle. Majorana looked for another solution: "How can we obtain a density independent nuclear mass without obstructing the free movement of particles by an artificial impenetrability?". His idea was to assume an exchange force which depends only on the position coordinates of a neutron and a proton and not on their spin. In modern terminology the interaction is written as

$$
V_{m}(r)=J(r) P_{m}
$$

where $P_{m}$ is the Majorana operator which exchanges the position of a proton and a neutron. This interaction is spin independent ${ }^{1}$ and, in the $\alpha$-particle, each proton can interact with 2 neutrons and vice-versa. The theory could account for the large binding of the $\alpha$-particle compared with that of the deuteron and ${ }^{3} \mathrm{He}$. Majorana wrote that, because of the Pauli principle "we may expect that, in

\footnotetext{
${ }^{1}$ In contrast to Majorana's interaction the Heisenberg force, in modern notation, has the form
}

$$
V_{H}(r)=J_{H}(r) P_{m} P_{\sigma}
$$

where $P_{\sigma}$ exchanges the spin of a proton and a neutron. This interaction has the opposite sign depending on whether the neutron-proton spins are parallel or anti-parallel. 
agreement with experiments, in heavy nuclei the mass defect per nucleon would not be noticeably bigger than the alpha particle". He wrote also that he would treat the neutron and proton as different particles and so "eliminate the troublesome $\rho$-spin coordinates". (The $\rho$ was Heisenberg's symbol for the isospin variable.) This was a big simplification and was very sensible at the time. Although Heisenberg had invented the isospin notation it did not correspond to any symmetry because he only considered neutron-proton forces.

Majorana showed that the high density limit the potential energy per nucleon is

$$
V_{\infty}=2 \frac{Z}{Z+N} J(0)
$$

if the proton number $Z$ is less than the neutron number $N$ with a similar expression if $Z>N$. Here $J(0)$ is the strength $J(r)$ of the neutron-proton exchange potential evaluated at $r=0$. The potential energy in Majorana's theory is negative and decreases monotonically as a function of density to a finite limit. The kinetic energy is positive and increases without limit with the density. Thus the total energy has a minimum. The strength and the range of the exchange force can be adjusted to reproduce the equilibrium binding energy and density of nuclear matter. Majorana's theory had solved the saturation problem without having to postulate a strong repulsion for small neutronproton separations. The theory was so satisfying aesthetically that it was accepted immediately and, with modifications, it was the basis for discussing nuclear binding energies for many years.

\section{Subsequent developments}

In 1936 Bethe wrote a series of three review articles on nuclear structure and reactions which are a useful reference even today. In the first of these [7] long sections were devoted to the nuclear force and to the structure of the deuteron, the $\alpha$-particle and heavier nuclei.

Bethe's paper contains many detailed calculations In section 20, he assumed a Gaussian radial form for the interaction and found sets of parameters which fitted the binding energies of the deuteron, ${ }^{3} \mathrm{He}$ and ${ }^{4} \mathrm{He}$ with only neutron-proton forces. The calculations used the Majorana exchange interaction and a variety of simple variational wave functions. Later in section 24 he gave results of calculations of nuclear matter with the statistical model (Hartree method) but found a problem: the binding energy was too small. In a similar way it was possible to find parameters which fitted the nuclear matter binding energy and density; but then the binding energy of the alpha particle comes out to be much too large. Thus a simple monotonic form for the radial dependence could not fit the binding energies of light nuclei and nuclear matter.

Evidence for exchange forces emerged in neutron-proton scattering experiments. Exchange forces interchange the nature of the particles. An incoming neutron which tends to continue in the forward direction becomes a proton and the neutron tends to be scattered in the backward direction in the center-of-mass system. Experiments at Berkeley [8] in 1949 showed a forward and a backward peak of similar magnitude which confirmed that the neutron-proton interaction contained a strong exchange component.

We know now that the nuclear force is much more complicated than the one assumed by Majorana. One very important qualitative result which emerged from nucleon-nucleon scattering experiments is the existence of a repulsive core in the nucleon-nucleon interaction. It was only in 
1951 that an interpretation of some experiments by Jastrow [9] suggested that Heisenberg's original idea of a hard core might be correct. Subsequent experiments showed that the proton-proton S-wave phase shift changes from positive to negative at about $250 \mathrm{MeV}$ laboratory energy. This result is inconsistent with a force which is attractive for all separations and shows that there must be a repulsive core with a radius of about $0.5 \mathrm{fm}$. This core is important, together with exchange forces, for explaining the saturation of nuclear forces.

In fact one should not try to fit the deuteron together with ${ }^{3} \mathrm{He}$ and ${ }^{4} \mathrm{He}$ because the tensor force makes an important contribution to the binding energy of the deuteron while in mean field theory it contributes nothing to the binding energy of ${ }^{3} \mathrm{He}$ and ${ }^{4} \mathrm{He}$. The existence of the tensor force was not known until much later. It was predicted by Yukawa (1938) and Kemmer (1938) and the prediction was verified by the measurement of the deuteron quadrupole moment by Kellog and his collaborators in 1939 [10].

\section{Majorana's legacy}

Majorana' most important legacy for the development of nuclear physics was his demonstration that symmetry of the nuclear force played a vital role in determining its effect on nuclear structure. His force and the one of Heisenberg made very different predictions about nuclear binding because of their different symmetry properties.

Another legacy was his use of the mean field method to calculate nuclear properties. During the past half-century the mean field method, both in its non-relativistic and relativistic forms, has been very important for calculating both dynamic and static effects in nuclei.

\section{References}

[1] G. Gamow, Atomic nuclei and radioactivity, The Clarendon Press, Oxford 1931.

[2] E. Majorana, On nuclear theory, Zeits. f. Phys., 82 (1933) 137;

Sulla teoria dei nuclei, La Ricerca Scientifica, 4 (1933), 550.

[3] W. Heisenberg, On the structure of atomic nuclei. I, Zeits. f. Phys., 77 (1932) 1.

[4] W. Heisenberg, On the structure of atomic nuclei. III, Zeits. f. Phys., 80 (1933) 587.

[5] H.C. Urey, F.G. Brickwedde and G.M. Murphy, A Hydrogen isotope of mass2 Phys. Rev., 50 (1936) 846.

[6] B. Cassen and E.W. Condon, On nuclear forces, Phys. Rev., 77 (1936) 846.

[7] H.A. Bethe and R.F. Bacher, Nuclear Physics, Rev. Mod. Phys. 8 (1936) 82.

[8] J. Hadley, E. Kelley, C. Leith, E. Segre, C. Wiegand and H. York, Experimentson n-p scattering with 90 and $40 \mathrm{MeV}$ neutrons, Phys. Rev. 75 (1949)351.

[9] R.Jastrow, On the nucleon-nucleon interaction, Phys. Rev. 81 (1951) 165.

[10] K.B.M. Kellog, I.I. Rabi, N.F. Ramsey and J.R. Zacharias, An electric quadrupole moment of the deuteron Phys. Rev., 50 (1936) 846. 